\title{
A Puzzle About Ontological Commitments
}

Philip A. Ebert

Philosophia Mathematica (III), 200816 (2), pages: 209-226

URL: http://dx.doi.org/10.1093/philmat/nkm050

This is a pre-copy-editing, author-produced PDF of an article accepted for publication in Philosophia Mathematica following peer review. The definitive publisher-authenticated version: Philosophia Mathematica (III), 200816 (2), 209-226 is available online at: http://dx.doi.org/10.1093/philmat/nkm050 


\title{
A Puzzle About Ontological Commitments
}

\author{
Philip A. Ebert*
}

September 17, 2007

\section{Introduction}

The aim of this paper is to scrutinise the necessary conditions for a specific mathematical principle to be ontologically committing and, as such, to identify the source of its ontological commitments. The principle in question is Hume's Principle - a statement that embedded in second-order logic allows for a deduction of the second-order Peano axioms. ${ }^{1}$ This principle is at the heart of the so-called Neo-Fregean programme as defended by Bob Hale and Crispin Wright. ${ }^{2}$ Once it is clear what the source of the commitment to infinitely many objects of Hume's Principle is, we should be able to re-evaluate the debate between the Neo-Fregeans - who defend Hume's Principle as an

\footnotetext{
${ }^{*}$ University of Stirling, Stirling, FK9 4LA, p.a.ebert@stir.ac.uk

${ }^{1}$ This theorem, known as Frege's theorem, was first explicitly noted by Parsons, in his (Parsons, 1965) and later independently rediscovered in (Wright, 1983), pp. 158-169. It was most likely known to Frege himself (see (Heck, 1993)). More recent presentations of the proof can be found in (Boolos, 1987a) (discursive), (Boolos, 1990) (rigourous), (Boolos, 1995), and (Boolos, 1996). Note that even a weaker version of Hume's Principle - Finite Hume - suffices for this derivation; see (Heck, 1997). Second-order logic is required for this proof. A relatively moderate portion of second-order logic suffices, however: $\Pi_{1}^{1}$ comprehension is enough. (Linnebo, 2004) has shown that Frege's Theorem cannot be proven in predicative second-order logic using Frege's own definition of the arithmetical primitives. (Heck, 2006) provides a proof that ramified second-order logic suffices. For a general overview of the technical details of Fregean arithmetic, see (Burgess, 2005).

${ }^{2}$ The main sources for a defence of the Neo-Fregean programme are (Wright, 1983), (Hale, 1987) and (Hale and Wright, 2001).
} 
analytic principle - and the so-called epistemic rejectionists ${ }^{3}$ - who deny its analytic status. The conclusions can then be generalised to other abstraction principles, principle that share a similar form to Hume's Principle. ${ }^{4}$

In the first section, I will clarify what epistemic rejectionism is committed to and provide a theoretical basis for the position by introducing the notion of presumptuousness as the underlying criterion on the basis of which Hume's Principle is to be rejected as an analytic principle. Then, I will review certain results given in (Shapiro and Weir, 2000) and in more formal detail by (Cook, 2003a) which prima facie put pressure on epistemic rejectionism. In section 4, I will propose a short thought-experiment to highlight the problem for epistemic rejectionism posed by the formal results and then suggest various responses on behalf of the epistemic rejectionist. The upshot will be to elicit a new and very basic disagreement between epistemic rejectionism and the $\mathrm{NeO}$ Fregeans which will provide a new angle to properly assess and re-evaluate the current debate.

\section{Boolos's epistemic rejectionism and the con- dition of presumptuousness}

Epistemic rejectionism repudiates the core idea of Neo-Fregeanism: Namely that certain mathematical principles - in particular certain abstraction principles - are analytic truths despite the fact that they have specific ontological commitments. Instead, the epistemic rejectionist claims that if a principle

\footnotetext{
${ }^{3}$ The position is labelled epistemic rejectionism since what it rejects is that HP is a truth that is knowable on analytic grounds. This is in contrast to rejectionism (simpliciter), a position that rejects the truth of HP if it is taken at face-value, i.e. as referring to numbers as abstract entities.

${ }^{4}$ An abstraction principle is of the following form:

$$
\forall \alpha \forall \beta(\xi(\alpha)=\xi(\beta) \leftrightarrow \alpha \approx \beta)
$$

where $\xi$ is a term-forming operator applicable to expression of the type of $\alpha$ and $\beta$ and $\approx$ is an equivalence relation on entities denoted by expressions of that type. Hume's Principle has the form of an abstraction principles (equinumerosity is the relevant equivalence relations). Frege's ill-fated Basic Law V is also an abstraction principle (involving co-extensionality as the relevant equivalence relation).
} 
has ontological commitments then it cannot be regarded as an analytic truth. So characterised, epistemic rejectionism adopts what Tennant once called the fourth dogma of empiricism for mathematical principles, i.e. "having existential commitments entails syntheticity." 5

In this paper, I will focus mainly on George Boolos' arguments and considerations. ${ }^{6}$ Boolos' specific formulation of the worry can be found in the suitably entitled paper "Is Hume's Principle analytic?" Importantly, his reflections do not only apply to Hume's Principle, but more generally to any abstraction principle and, for that matter, to any axiom or implicit definition that has existential commitments.

Boolos' view is that statements that are analytic involve (at least) two components: "Firstly, they are true; secondly and roughly speaking, they lack content, i.e. they make no significant or substantive claims or commitments about the way the world is; in particular, they do not entail the existence either of particular objects or of more than one object." 7 More precisely, and concerning Hume's Principle (HP), Boolos continues: "The main significant worry for the defender of the analyticity of HP concerns the quite strong content that it appears to possess." 8 Namely, "if HP holds [...] there must be infinitely many objects" ${ }^{9}$, and so he concludes: "[HP] taken as an axiom, might then entail that, for example there are many, many objects, too many for it to be capable of being regarded any longer as analytic." 10

Importantly, however, we need to ask under what conditions does Hume's Principle have a commitment to infinitely many objects and which component of the principle is it that generates these commitments? Standardly, in order to discern the ontological commitments of a theory or a principle, one

\footnotetext{
${ }^{5}$ (Tennant, 1997), p. 303. It is, of course, also a dogma of Kantian Philosophy.

${ }^{6}$ See especially (Boolos, 1997). There are similar thoughts in his earlier papers, for example (Boolos, 1987a), (Boolos, 1987b) and (Boolos, 1990). In some his papers, Field adopts what I label epistemic rejectionism. See for example his review article (Field, 1984) of (Wright, 1983) - however, Field is here not only concerned with analyticity but he also rejects the thought that ontologically committing mathematical principles are a priori knowable.

${ }^{7}$ (Boolos, 1997), p.303

${ }^{8}$ op. cit., p. 304

${ }^{9}$ op.cit., p. 306

${ }^{10}$ op. cit., p.309, my italics
} 
consults the corresponding Ramsey sentence(s) of the theory or the principle in question to read off its ontological commitments. So, in the case of Hume's Principle, we get the following statement:

Ramsified Hume's Principle (RHP)

$\exists \eta \forall f \forall g(\eta f=\eta g \leftrightarrow f \sim g)^{11}$

That is, Hume's Principle commits one to the existence of a higher-order function - an entity, whichever it is that maps concepts on to objects, giving distinct values for each pair of non-equinumerous concepts. So, it may seem that in the case of Hume's Principle it is the existence of this function which is problematic and can't be assumed on purely analytic grounds since it is this function that involves an implicit commitment to infinitely many objects. This seems to be exactly what Boolos has in mind in the following passage:

Our present difficulty is this: just how do we know, what kind of guarantee do we have, why should we believe, that there is a function that maps concepts to objects in the way that the denotation of octothorpe [the symbol Boolos used to denote the number function] does if HP is true? If there is such a function then it is quite reasonable to think that whichever function octothorpe denotes, it maps non-equinumerous concepts to different objects and equinumerous ones to the same object, and this moreover because of the meaning of octothorpe, the number-of-sign or the phrase "the number of". But do we have any analytic guarantee that there is a function that works in the appropriate manner? ((Boolos, 1997), p. 306, my italics)

Thus, Boolos believes that it is, we might say, presumptuous to assume that we can have an analytic guarantee - and with this I presume he means a justification on purely analytic grounds - for the existence of such a function since it has very specific demands on the object level. In particular, in the case of Hume's Principle, the relevant function commits one to the existence

\footnotetext{
${ }^{11}$ ' $\eta$ ' is a variable ranging over functions from properties to objects and ' $\mathrm{f} \sim \mathrm{g}$ ' means that there is a one-to-one relation between the f's and the g's.
} 
of too many objects. Thus, as a reconstruction of Boolos' thought and so of the epistemic rejectionist in general, we can put forward the following general criterion of presumptuousness:

\section{Criterion of Presumptuousness}

Assuming on a purely analytic basis the existence of a function is presumptuous, if and only if its application has further ontological commitment on the object level.

In order to be more precise we now have to clarify the notion of further ontological commitment. Boolos' worry about the commitments of Hume's Principle concern the demands on the cardinality of the world for this abstraction principle to be true. For, as Boolos says, if Hume's Principle is true, there will have to be infinitely many objects and thus there will have to be "many, many objects, too many for it to be capable of being regarded as analytic." 12 As a result, we can best interpret him adopting the following notion of further ontological commitment:

\section{Further Ontological Commitment}

A function has further ontological commitment if the assumption of its existence commits one to the existence of at least $\kappa$-many objects in the first-order domain, whereas $\kappa$ is such that it has at least cardinality $\aleph_{0} .{ }^{13}$

So, we now have a notion of presumptuousness and further ontological commitments to underwrite the epistemic rejectionist complaint that the NeoFregean presumptuously assumes the existence of the function referred to by the number-operator in Hume's Principle. On this conception, Hume's Principle clearly is presumptuous as it entails the existence of infinitely many

\footnotetext{
12 op. cit., p.309

${ }^{13}$ Note that there might well be other interpretations of the idea of further ontological commitments. Maybe $\kappa$ should be chosen to be finite or so that even one object is too many in Boolos' sense and thus presumptuous. We will discuss other interpretations, which depart from Boolos' original idea in section 5. It is also noteworthy here that this interpretation of Boolos assumes the axiom of (countable) choice, so that there being too-many, i.e. infinitely many objects entails there being $\aleph_{0}$-many objects.
} 
objects and thus $\aleph_{0}$-many objects. Since, according to Boolos, no analytic principle can itself commit one to the existence of that many things, Hume's Principle can't be an analytic truth. I believe that it is this notion of presumptuousness together with the just characterised notion of further ontological commitments that best captures Boolos' original complaint.

In the following, I want further to explore the epistemic rejectionist's thought by discussing different background assumptions. As noted by Hale and Wright on various occasions ${ }^{14}$, Hume's Principle itself does not entail the truth of instances of the right-hand side; rather, it is only in conjunction with further - presumably necessary - truths that Hume's Principle entails the existence of infinitely many objects. In the next section, I want to exhibit how crucial this additional component is, and that it is not without additional non-trivial assumptions that Hume's Principle is ontologically committing. To this end, I will briefly discuss two different metaphysical pictures giving rise to two different comprehension schemes which, as I will show, affect the presumptuousness of the introduction of the operator involved in Hume's Principle.

\section{Different comprehension schemes}

There have been a number of previous discussions of aristotelian logic and Hume's Principle. ${ }^{15}$ Hume's Principle is a statement in second-order logic which involves higher-order quantification. Higher-order quantification itself raises various questions and here we need to distinguish a number of interrelated issues pertaining to the domain of quantification of the higher-order quantifiers.

One issue is what kinds of entities are in the domain of the higher-order quantifier, another is how many of these entities are there and lastly, what does it take to be in the domain of the higher-order quantifers. Leaving

\footnotetext{
${ }^{14}$ See e.g. (Hale and Wright, 2000), p. 146 (Wright, 1990) (p.162f) and (Hale, 1994) (p.184) (as quoted from (Hale and Wright, 2001))

${ }^{15}$ It is first mentioned in (Shapiro and Weir, 2000) and recently worked out in formal details in (Cook, 2003a).
} 
aside the first question and thus issues raised by Quine, Boolos and many others $^{16}$, let us grant the existence of properties as the entities that higherorder quantifiers range over and focus on the second and third issue. In the context of this paper, I won't argue for a specific answer to these questions; rather I would like to note that two traditional positions on what it takes for a property to exist can be distinguished, which leads to different conceptions of how many properties there are.

We can distinguish an aristotelian conception from a platonist conception. What it takes for a property to exist, according to the aristotelian, is that the property has to be in rebus. Roughly speaking, properties are concrete universals that exist provided that they are exemplified by a concrete object. The platonist, in contrast, has no such restriction, rather properties are ante rem and their existence does not depend on whether they are concretely instantiated or not.

As a result, how many properties one thinks there are depends upon the metaphysical framework that is adopted. For the platonist - translated into modern philosophers' "slang" - standard comprehension is true, while the aristotelian, on the other hand, insists on a modification of the standard comprehension scheme, since only those properties that are instantiated (in rebus) exist. So, the modern platonist embraces the following unconditional comprehension scheme:

Full comprehension scheme (FCS)

$\exists F \forall x_{1}, x_{2}, \ldots, x_{n}\left(F x_{1}, x_{2}, \ldots, x_{n} \leftrightarrow \phi x_{1}, x_{2}, \ldots, x_{n}\right)$

while the modern aristotelian would embrace the following conditional comprehension scheme:

Aristotelian comprehension scheme $(A C S)^{17}$

\footnotetext{
${ }^{16}$ See (Quine, 1986) for his notorious "Wolf in sheep's clothing" - phrase and (Boolos, 1985), and (Boolos, 1984) for Boolos' suggestion of plural interpretation of the secondorder quantifiers. See also (Rossberg, 2006) for defending the view that higher-order quantification does not involve any kinds of entities.

${ }^{17}$ There are various ways of characterising the aristotelian comprehension scheme. An interesting question is whether the aristotelian should be characterised as an agnostic about the empty property, rather than a believer in the non-existence of this property. Here I won't dwell on the agnostic characterisation.
} 


$$
\begin{aligned}
& \exists x_{1}, x_{2}, \ldots, x_{n} \phi x_{1}, x_{2}, \ldots, x_{n} \rightarrow \exists F \forall x_{1}, x_{2}, \ldots, x_{n}\left(F x_{1}, x_{2}, \ldots, x_{n} \leftrightarrow\right. \\
& \left.\phi x_{1}, x_{2}, \ldots, x_{n}\right)
\end{aligned}
$$

Let us assume that both positions are genuine positions in metaphysics in that each conception can be sufficiently motivated (at least from a neutral position). Also, let us assume that these different metaphysical pictures can lead to the adoption of different comprehension schemes. A platonist will adopt standard comprehension, while an aristotelian will adopt a different comprehension scheme since his existence criterion for properties is constrained to those that are instantiated. What I will show in the next section, is that these two distinct positions that are reflected in two different comprehension schemes have a major effect on the issue of presumptuousness.

\section{Hume's Principle within an aristotelian framework}

First, we need to note the interesting result that the standard proof for the existence of infinitely many numbers on the basis of Hume's Principle does not go through without the existence of the empty property. Intuitively, this is because within the aristotelian framework it can't be proven that there are $n+1$ numbers less than or equal to $n .{ }^{18}$ When embedded in a logic with an aristotelian comprehension scheme, Hume's Principle hardly entails any additional ontological commitments. Cook $^{19}$ shows that if there is a non-empty universe, then Hume's Principle only entails the existence of the number 1 (but not 0), otherwise Hume's Principle in an aristotelian setting is satisfiable on the empty domain.

Having this result in place, how does it affect our previous discussion of the presumptuousness of Hume's Principle? Adopting the conception of further ontological commitment outlined before and thus regarding commitments as critical once they are too many in the sense of at least $\aleph_{0}$-many, Hume's Principle, with respect to the aristotelian comprehension, is not pre-

\footnotetext{
${ }^{18}$ See especially (Shapiro and Weir, 2000), p.168 where this result is further explained.

${ }^{19}$ In his (Cook, 2003a)
} 
sumptuous! All Hume's Principle will give us, in this setting, is an additional commitment to the number 1, i.e. to one more object; it will never inflate an originally finite domain to an infinite one. So, assuming I correctly reflected Boolos' thoughts, the epistemic rejectionist seems committed to regard Hume's Principle, if embedded in an aristotelian framework, as non-presumptuous. It does not involve the existence of too many objects, i.e. $\aleph_{0}$-many objects.

This result is already interesting since it further elaborates, and gives credence to, the thought that it is not Hume's Principle itself that entails the existence of infinitely many objects; rather additional assumptions regarding which properties exist have to be in place. ${ }^{20}$ The following section will introduce a puzzle about the ontological commitments of Hume's Principle based on these formal results which puts pressure on the epistemic rejectionist position and their conception of presumptuousness.

\section{A puzzle about Hume's Principle}

Consider the following story:

"Imagine a young upcoming logician/philosopher, well-trained and a committed epistemic rejectionist, who thinks that it is impossible to have a purely analytic guarantee for any principles that involves a presumptuous function. However, our logician/philosopher - call him George - is also an aristotelian, thus he thinks that only properties exist that are instantiated. Well, now let George come across Hume's Principle (as a good logician should); having

\footnotetext{
${ }^{20}$ Shapiro and Weir (see their (Shapiro and Weir, 2000) especially p.168ff.) makes it seem as if the empty property is what is responsible for the ontological commitments. They write: "The neo-logicist cannot claim, then, that the existence of infinitely many numbers is demonstrable in an epistemically innocent fashion unless he or she can show that it is demonstrable, in such an epistemically innocent fashion, that there is an uninstantiated property. [...] The 'aristotelian neo-logicist' will, it is true, be able to prove $(\exists F \forall y \neg F y \rightarrow$ Inf $)$

where Inf is a theorem of infinity; that is, if an empty property exists then there are infinitely many numbers." (op.cit) The following discussion will, I hope, clarify the points raised in their discussion.
} 
studied it, he does not have any complaints about Hume's Principle at least none on the grounds of the constraint of presumptuousness. Thus, assuming the principle fulfils other constraints which are necessary for its success (and assume that nothing else stands in the way of regarding it as analytic) George is content to claim to know Hume's Principle as an analytic principle.

Now, as it happens George visits St. Andrews one day, encounters some philosophers there and by talking to them, he becomes convinced that he should allow the empty property within his metaphysics. That is he changes his metaphysics of properties from an aristotelian conception to a platonist one.

But George has been set up by those philosophers. Having changed his metaphysical framework, they challenge him to explain how his change is compatible with his position as a committed epistemic rejectionist and his previous endorsement on purely analytic grounds of Hume's Principle.

The problem thus is that George has previously accepted the existence of the number-function (i.e. the truth of the Ramsey-sentence of HP) while accepting aristotelian metaphysics. But by adopting a different metaphysical conception (whose only relevant difference is that it involves the existence of one more property), the number function picked out by Hume's Principle has become presumptuous!"

So what is George to do? Let me outline the above puzzle in more detail and develop it as a challenge to, or rather reductio of, epistemic rejectionism. This is followed by a discussion of different lines of reply to this challenge.

\section{Premise 1}

The existence of the number-function picked out by Hume's Principle in aristotelian logic is not presumptuous, and thus (assuming it fulfils other relevant criteria) is acceptable on analytic grounds. 


\section{Premise 2}

Changing the underlying metaphysics from aristotelian to nonaristotelian does not change the analytic grounds for accepting a principle and it is in this respect an innocuous change.

\section{Conclusion}

As the existence of the function, denoted by the number-operator is already accepted on analytic grounds, a rejectionist is committed to allowing the analytically justified claim of the existence of infinitely many objects.

and the underlying major premise of this reasoning is:

\section{Major Premise}

The nature of the number-function is independent of the existence of the empty property, i.e. making such justified changes in the metaphysical picture has no bearing on the identity of the number-function.

In the following, I will discuss the different claims of the argument in turn and suggest various replies that can be made to each premise. ${ }^{21}$

\subsection{Rejecting premise 1}

One line of response is to reject the first premise. Here, one might doubt this claim on the grounds that appreciating that Hume's Principle is presumptuous in a platonistic metaphysics shows that it is presumptuous tout court. In

\footnotetext{
${ }^{21}$ One referee observed that the full ontological commitments of HP (i.e. commitments to infinity) re-emerge even on an Aristotelian framework provided one has an a priori proof of the existence of at least one non-number, or alternatively, if one adopts certain modal consideration. This type of reasoning is not without its own presuppositions (Caesartype worries about the former, the relevant strength of modal considerations (S5) on the other). Furthermore, we could add further twists to the initial story to neutralise such undermining assumption, which is why I will not further discuss them here. Nevertheless, his observations point to the fact that changing other background assumptions can have similar results even within the aristotelian setting. We could, for example, easily create another puzzle: have George be an Atheist and then later, on becoming a Theist - while retaining his aristotelian comprehension scheme - he comes to acknowledge new ontological commitments of HP! I won't discuss this puzzle here.
} 
both definitions (that is Hume's Principle embedded in aristotelian and embedded in platonist comprehension) the number operator refers to the same function, only that its occurrence in the platonist framework shows that the principle is presumptuous.

This line of reply, however, is unattractive if the sole reason for George to give up Hume's Principle is on the ground of its "potential" presumptuousness. It would seem that rejecting the first premise on this basis in order to avoid the puzzle presupposes that the platonist conception has a somehow higher priority over the aristotelian framework in evaluating the ontological commitments of a principle. Consequently, what matters concerning the notion of presumptuousness would have to be rephrased in order to properly reflect the epistemic rejectionist's reasoning, if this strategy were pursued, such that:

\section{Strong condition of presumptuousness}

Assuming on a purely analytic basis the existence of a function is presumptuous, if and only if its application has further ontological commitment on the object level in a platonist comprehension scheme.

This change of the condition of presumptuousness is clearly unattractive since it demands that even a committed aristotelian should take into account the possibility of further ontological commitments in the platonist framework which, one might well have supposed, should not matter to him. Therefore, rejecting the first premise is not an attractive strategy.

\section{$4.2 \quad$ Rejecting premise 2}

The second premise comprises the claim that a change from an aristotelian to a platonistic metaphysics of properties is innocuous with respect to the analytic grounds for accepting a principle. This claim can obviously be challenged in various ways. Let us here first scrutinise what exactly a change of metaphysical framework involves. 
What might well be called an 'inconsequential' change in our metaphysical conception would involve merely the quantity of properties that one assumes to exist. On the platonistic conception for example, there exists one more property - the empty property - that doesn't feature in the aristotelian conception. One view then, is that the aristotelian properties are of the same kind as the platonistic ones; the two frameworks just differ in terms of what properties that are out there.

In contrast, a more substantial understanding of the change takes into account the kind of motivation for adopting one conception over the other. As mentioned before, an aristotelian and a platonist differ in what it takes for a property to exist. While the former considers properties as concrete universals, existing in rebus, the platonist conceives of properties as pure universals existing as abstract entities. This difference might then not only give rise to a different answer to the question of how many properties are there, but also might lead one to suppose that the kinds of properties are substantially different in the different metaphysical settings. Therefore, since there are different kinds of properties involved in the different metaphysical views merely changing the comprehension scheme is not enough to accurately reflect the change: Different kinds of properties bring with them a different senses for principles such as Hume's Principle, i.e. the sense of the numberoperator is substantially different in both cases. On this basis, one may argue that a transition from an aristotelian to a platonistic framework is not innocuous: it might well be that the "analytic grounds" will not be the same on the different conceptions since the sense of the number-operator changes. In turn, this result could be used as a motivation to reject the Major Premise, and so argue that different functions are being picked out by the expression "the number of" within the different metaphysical frameworks - different, since they have different kinds of entities as arguments.

So, an important lesson - a lesson that is missing from the discussion of (Shapiro and Weir, 2000) and (Cook, 2003a) - is that unless one looks more closely at the views underlying the respective comprehension schemes, many crucial metaphysical issues remain untouched. However, is the possibility of the 'substantial reading' of change enough to defuse the puzzle? 
I think the following formal move can be used to re-instantiate the puzzle, at least in the case of Hume's Principle: Instead of conceiving of the number operator as denoting a function from properties to numbers, one could regard it as a function from the Fregean extensions of properties to numbers (thereby relying, to be sure on a suitably restricted version of Basic Law $\mathrm{V}^{22}$ ). Presumably, even if one should regard platonist properties as distinct in kind from aristotelian properties, the extensions of such properties do overlap. To use an example, we may grant that the platonistic property which holds just of Hale and Wright is distinct from the aristotelian property which holds just of Hale and Wright, as the former is ante rem while the latter is in rebus. Nonetheless, the extension of the former property is identical to the extension of the latter and so the worry about the substantiality of the transition from aristotelian conception of properties to a platonistic conception should not get a grip here. The function involved in this revised version of Hume's Principle is a function from objects to objects and so, the substantiality of a change in one's metaphysics of properties, does not have a bearing on whether this function from objects to objects is the same.

Before tackling the issue raised in the Major Premise, i.e. whether the function (be it the standard version or the above revised version) may be viewed as denoting the same function in both aristotelian and platonist comprehension schemes ${ }^{23}$, let me discuss a further attempt to challenge premise 2 .

Another way to reject the premise is to point out that whereas in the aristotelian setting George knows, or at least is in a position to know, HP as an analytic truth, he is surely not - in a platonist setting - in a position to know HP on purely analytic grounds, for the fact that HP is presumptuous will act as a defeater to his (presumed) knowledge of HP. Therefore, a change in the metaphysical setting is non-innocuous with respect to the analytic grounds since the change of metaphysical setting will undermine George's previous knowledge of HP as an analytic truth. Thus - so this reply goes

\footnotetext{
${ }^{22}$ As, for example, suggested by (Boolos, 1989) or (Cook, 2003b)

${ }^{23}$ If the above formal trick is used the Major Premise has to be suitably revised: The issue becomes whether the identity of the number-function is affected by the (non-) existence of the extension of the empty property.
} 
- the very fact that George cannot be credited with knowledge of HP as an analytic truth within the platonist setting undermines the viability of premise 2.

Various points can be made in response to this concern. First, we need to be clear what principle it is that he fails to know - or looses his knowledge of - when the metaphysical setting changes. On the assumption that the Major Premise does not hold, $\mathrm{HP}_{\text {aristotelian }}$ and $\mathrm{HP}_{\text {platonist }}$ will pick out different functions. Thus, it can then be argued that premise 2 holds, since, even in a platonist framework, George is still in a position to know $\mathrm{HP}_{\text {aristotelian }}$ on analytic grounds, whereas he is not in a position to know $\mathrm{HP}_{\text {platonist }}$ on analytic grounds - hence a change from an aristotelian to a platonistic metaphysics of properties is innocuous with respect to the analytic grounds for accepting a specific principle [namely $\mathrm{HP}_{\text {aristotelian }}$. If however, the Major Premise does hold, then the above considerations against premise 2 might gain some support since both $\mathrm{HP}_{\text {aristotelian }}$ and $\mathrm{HP}_{\text {platonist }}$ will pick out the same number-function yet it is the status as an analytic truth of the latter that is defeated by the presumptuousness of the principle. ${ }^{24}$ What this shows is that again much will hinge on the acceptance (or non-acceptance) of the Major Premise and it is this crucial premise that I will discuss in the next section. $^{25}$

\subsection{Rejecting the Major Premise}

The standard conception for the identity-conditions of functions spells matters out extensionally. So, the identity-conditions of a function depend upon the entities that do figure as arguments and values of the function. On the standard view, if two functions have the same inputs but provide different outputs, then the functions are distinct. Thus, the epistemic rejectionist could maintain that the number-function from properties to objects - or, the

\footnotetext{
${ }^{24}$ Whether or not this is sufficient to reject premise 2 is debatable. To evaluate this it would have to be clarified exactly what it is that is part of the "analytic grounds" for accepting a principle and to what extent they have to figure as part of the reason for George to accept a principle. This is a discussion that goes beyond the scope of this paper.

${ }^{25} \mathrm{I}$ 'm very grateful for comments of an anonymous referee on this section.
} 
number-function from extensions of properties to objects - in an aristotelian setting, is a different function from the one embedded in a platonistic set-

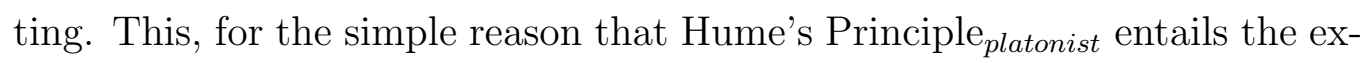
istence of infinitely many objects, while the latter Hume's Principle aristotelian $_{\text {n }}$ does not. This observation can then motivate the rejection of the Major Premise and gives credence to the thought that different entities are denoted by the number-operator occurring in Hume's Principle Principle ${ }_{\text {aristotelian }}$.

However, one motivation for thinking that the Major Premise holds, is to think of identity-conditions for functions non-extensionally. Think of the operator involved in Hume's Principle in analogy to a "vending machine". Given a certain input (properties) the vending machine (Hume's Principle) gives you a certain output (objects). But the peculiarity about this particular "machine" - the number-function involved in Hume's Principle - is that by giving it the "magic first coin" (the empty property) it not only returns you the "product" (number), but also guarantees that anytime this is repeated with a different "coin", a further new "coin" is issued, which enables you to do the same again and again, so to get infinitely many "products".

However, and here the analogy becomes relevant to the issue at hand, no matter what "coins" are given to the machine - whether you start with the magic one or not - the nature and constitution of the machine is the same. So, spelling out this metaphor, if we accept Hume's Principle embedded within aristotelian logic we encounter the same function as in the platonist case. The coins (properties) themselves and the amount of coins (properties) may change from one metaphysical setting to another but the nature of the machine (Hume's Principle) stays the same. Hume's Principle platonist $_{\text {involves }}$ the same function and indeed is the same principle as Hume's Principle aristotelian, $_{\text {, }}$ although different consequences can be deduced in the different metaphysical settings.

Do these considerations help us to see how we should conceive of the dialectic between the epistemic rejectionist on the one hand and the NeoFregean on the other? I will draw together the major points and diagnose in what position we now find ourselves. 


\section{$5 \quad$ Diagnosis: Rejectionism rejected?}

The puzzle about the ontological commitments of Hume's Principle was introduced to make the point vivid that Hume's Principle, and other abstraction principles, do not commit to objects all by themselves; rather other components have to be in place. The puzzle was used to put pressure on the epistemic rejectionist view and the notion of presumptuousness as characterised above. Hume's Principle embedded in aristotelian logic is not presumptuous ${ }^{26}$ so, if it is assumed that the identity of the function involved in Hume's Principle is invariant with respect to a change of the underlying comprehension scheme, the principle should also be non-presumptuous in a platonist framework. This conclusion could then be regarded as a reductio of the notion of presumptuousness presupposed by epistemic rejectionism.

We have seen that there is at least one way to avoid this problematic conclusion for the epistemic rejectionist, namely by denying the Major Premise (i.e. the invariance claim) on the basis of an extensional conception of properties. Here, I won't be able to foreclose this way out for the rejectionist. Thus, a main issue for further discussion remains the question whether the identity of functions should be characterised extensionally or not. If there are independent reasons for a non-extensional treatment, then the force of the puzzle is reinstated and the threat for the viability of epistemic rejectionism will return. ${ }^{27}$ Furthermore, we should also appreciate the more general lesson noted above, that focusing exclusively on Hume's Principle per se (and so abstraction principles simpliciter) in the discussion of ontological commitments is at least potentially confusing, at worst wrong! We should always keep in mind the necessary presuppositions, which go beyond mere logical reasoning; instead, as I argued, they are to be located within the metaphysics

\footnotetext{
${ }^{26}$ Pace the considerations mentioned in fn 21.

${ }^{27}$ If that is the right analysis, it seems that we can put further, at least prima facie, pressure on an extensional treatment of functions. Consider for example the Direction Abstraction: Should it really matter to the identity of the direction-function how many lines there are? I think not, still I will here have to leave a fuller investigation of this next round of discussion to another occasion. Note also, that if the Major Premise is to hold, it might be more tempting for the epistemic rejectionist to reject premise 2 as mentioned in the previous section. Again, I will have to leave a more detailed discussion of this further epicycles to another occasion.
} 
of properties and the metaphysics of logic.

Stepping back a bit from the details of the above puzzle, another lesson to draw from the discussion might be to reconsider, in more detail, the notion of further ontological commitments as extracted from Boolos' quotations. The initial interpretation is, I think, closest to Boolos' conception and concerns the commitment to infinitely many (i.e. too many) objects by accepting Hume's Principle. However, maybe a different characterisation can be independently motivated which would avoid the problematic result of classifying Hume's Principle embedded in an aristotelian comprehension scheme as nonpresumptuous and presumptuous in a platonist comprehension scheme. For example, the following notion of further ontological commitments would have the desired result:

\section{Further Ontological Commitment II}

A function has further ontological commitment if the assumption of its existence commits one to the existence of more objects on any domain.

So, the idea is that, whenever a function commits one to the existence of one or more objects on an arbitrary domain, then it has further ontological commitments. In particular, Hume's Principle embedded in an aristotelian setting would have further ontological commitments since it commits one to the existence of one more object (namely 1) on any non-empty domain. Thus, on this conception of further ontological commitments, the above puzzle would not get off the ground since Hume's Principle would be presumptuous in both the aristotelian and platonist setting. However, this notion is not what Boolos himself had in mind and another story will have to be told to motivate this conception.

Further, there are other interesting conceptions of the notion of further ontological commitments. For example, another view might take into account the kinds of objects that are introduced. So, rather than merely focusing on the effects on the cardinality by adopting Hume's principle, one might be concerned about the new kinds of objects - namely numbers - that are introduced by Hume's Principle. This discussion, however, would presuppose 
a solution to the well-known Caesar problem and the not so well-known $C-R$ problem. ${ }^{28}$ Again, adopting such a conception of further ontological commitments might, in the end, help the epistemic rejectionist to diffuse the above puzzle. Here, I can only indicate different possible ways to characterise this crucial notion underlying the rejectionist conception and will leave a critical discussion to a further occasion.

To conclude, I think that a fair evaluation of the status belli between the epistemic rejectionist and the Neo-Fregean is that it has reached a stalemate. Epistemic rejectionism, if characterised in terms of the adoption of the additional constraint of presumptuousness that I outlined in the beginning, will happily embrace an extensional conception of operators and functions, while his opponent will be tempted to accept a non-extensional conception. And while the former will accept the fourth dogma of empiricism, the latter will see the case of mathematics as a reason to reject it - another case of "one man's modus ponens is another's modus tollens" 29. Despite this lack of conclusively rejecting either Neo-Fregeanism or epistemic rejectionism, I hope, however, to have clarified, structured and advanced this discussion and pointed towards future avenues of how this debate should be pursued..$^{30}$

\footnotetext{
${ }^{28}$ This is a special version of the Caesar problem which might be left untouched by a solution to the usual Caesar problem. For a discussion of the $C$ - $R$ problem, see (Cook and Ebert, 2005)

29 (Boolos, 1997), p.308

${ }^{30}$ I wish to thank the audience of various Arché Seminars in St Andrews for their comments and discussion. Special thanks to Roy T. Cook, Marcus Rossberg and Crispin Wright for commenting and discussing earlier drafts of this paper. Also, I wish to thank two anonymous referees for their very helpful comments.
} 


\section{References}

Boolos, G. (1984). To be is to be a value of a variable (or to be some values of some variables). The Journal of Philosophy, 81:430-49. Reprinted in George Boolos, Logic, Logic and Logic.

Boolos, G. (1985). Nominalist platonism. Philosophical Review, 94:327-44. Reprinted in George Boolos, Logic, Logic and Logic.

Boolos, G. (1987a). The consistency of Frege's foundation of arithmetic. in J.J. Thomson, ed., On Being and Saying: Essays for Richard Cartwright, p.3-20, Cambridge, Mass.:The MIT Press. Reprinted in George Boolos Logic, Logic and Logic.

Boolos, G. (1987b). Saving Frege from contraction. Proceedings of the Aristotelian Society, 87:137-51. Reprinted in George Boolos, Logic, Logic and Logic.

Boolos, G. (1989). Iteration again. Philosophical Topics, 42:5-21. Reprinted in George Boolos, Logic, Logic and Logic.

Boolos, G. (1990). The standard of equality of numbers. In George Boolos, Meaning and Method: essays im honor of Hilary Putnam, Cambridge: Cambridge University Press. Reprinted in George Boolos, Logic, Logic and Logic.

Boolos, G. (1995). Frege's theorem and the Peano postulates. The Bulletin of Symbolic Logic, 1:317-26. Reprinted in George Boolos, Logic, Logic and Logic.

Boolos, G. (1996). On the proof of Frege's theorem. In A. Morton and S. Stich (eds) Benacerraf and his Critics, Oxford: Blackwell. Reprinted in George Boolos, Logic, Logic and Logic.

Boolos, G. (1997). Is Hume's Principle analytic? In Richard Heck (ed), Language, Thought and Logic. Reprinted in George Boolos, Logic, Logic and Logic. 
Boolos, G. (1998). Logic, Logic and Logic. Harvard, Cambridge, Massachusetts.

Burgess, J. P. (2005). Fixing Frege. Princeton University Press, Princeton.

Cook, R. T. (2003a). Aristotelian logic, axioms and abstraction. Philosophia Mathematica, 11.

Cook, R. T. (2003b). Iteration one more time. Notre Dame Journal of Formal Logic, 44(2):63-92.

Cook, R. T. and Ebert, P. A. (2005). Abstraction and identity. Dialectica: Special Edition on the Julius Caesar Problem, 59(2):1-19.

Field, H. (1984). Platonism for cheap? Crispin Wright on Frege's context principle. Canadian Journal of Philosophy, 14:637-662. reprinted in Hartry Field Realism, Mathematics and Modality.

Hale, B. (1987). Abstract Objects. Basil Blackwell, Oxford.

Hale, B. (1994). Dummett's critique of Wright's attempt to resusciate Frege. Philosophia Mathematica, 3:122-47. Reprinted in Bob Hale and Crispin Wright, The Reason's Proper Study.

Hale, B. and Wright, C. (2000). Implicit definition and the a priori. In Paul Boghossian and Christopher Peacocke (eds.), New Essays on the A Priori; reprinted in Bob Hale and Crispin Wright, The Reason's Proper Study.

Hale, B. and Wright, C. (2001). The Reason's Proper Study: Essays towards a Neo-Fregean Philosophy of Mathematics. Clarendon Press.

Heck, R. (1993). The Development of Arithmetic in Frege's Grundgesetze der Arithmetik. Journal of Symbolic Logic, 58:579-601.

Heck, R. (1997). Finitude and Hume's Principle. Journal of Philosophical Logic, 26:589-617. 
Heck, R. G. (2006). Ramified Frege Arithmetic. Journal of Philosophical Logic. Forthcoming; available online: $<$ http://phonline.org/paper.php?keynum $=522>$.

Linnebo, Ø. (2004). Predicative Fragments of Frege Arithmetic. Bulletin of Symbolic Logic, 10:153-174.

Parsons, C. (1965). Frege's theory of numbers. In Max Black (ed), Philosophy in America, (Ithaca:Cornell University Press, p. 180-203).

Quine, W. V. (1986). Philosophy of Logic, 2nd edition. Harvard, Cambridge, Massachusetts.

Rossberg, M. (2006). In Defence of Second-order Logic. PhD dissertation, University of St Andrews.

Shapiro, S. and Weir, A. (2000). 'Neo-Logicist' logic is not epistemically innocent. Philosophia Mathematica, 8(3):160-189.

Tennant, N. (1997). The Taming of the True. Oxford University Press, Oxford.

Wright, C. (1983). Frege's Conception of Numbers as Objects. Aberdeen University Press, Aberdeen.

Wright, C. (1990). Field and Fregean platonism. In Andrew Irvine, Physicalism in Mathematics, Kluwer, Dordrecht/Boston/London, p. 73-93 reprinted in Hale/Wright The Reason's Proper Study. 